\title{
La participación social en la escuela en México: ¿un problema de democracia y cultura política?'
}

\author{
Social participation in schools of Mexico: \\ ¿Is there a problem in terms of democracy and political culture?
}

\section{A participação social na escola do México: um problema de democracia e cultura política?}

\section{Pedro Flores-Crespo*}

\section{Resumen}

Este artículo cuestiona la tesis culturalista para explicar porqué la participación social no ha podido surgir en el sector educativo de México. Basado en un trabajo empírico con escuelas secundarias de tres estados: Chiapas, Ciudad de México y Durango ( $n=27)$, este trabajo muestra que los individuos sí muestran predisposición a intervenir de manera crítica y razonada en sus centros escolares, no obstante, no existen las estructuras de oportunidad para ampliar competencias ciudadanas, esos mismos actores no parecen verse como iguales y hay entendimiento superficial del mundo juvenil por parte de los maestros que impiden que la participación del ciudadano se despliegue, pese al régimen autoritario construido por el sistema de partido único (1920-2000).

Palabras claves: Igualdad. México. Participación ciudadana. Secundaria.

\section{Abstract}

this article calls to question the idea that social participation and parental engagement cannot flourish in Mexico due to an authoritarian path dependency. This idea is discussed by using empirical evidence of upper secondary schools in three states of Mexico: Chiapas, Mexico City and Durango $(n=27)$. Irrespectively of the context, students exercise somewhat their democratic capacities. However, real opportunities for enlarging these capacities are limited. Students are not seen as equals by teachers. Mentors, in addition, tended to underestimate young people attitudes. In short, citizenship is emerging but school conditions have restrained such democratic reasoning (1920-2000).

Keywords: Deliberative democracy. Mexico. Citizen engagement. Upper secondary school.

Recebido em 27/02/2019 - Aprovado em 23/06/2019

http://dx.doi.org/10.5335/rep.v26i3.9446

Profesor-investigador de la Universidad Autónoma de Querétaro (FCPyS). México. ORCID: 0000-0002-5508-1464. E-mail: pedro.florescrespo@uaq.mx 


\title{
Resumo
}

\begin{abstract}
Este artigo questiona a tese culturalista, para explicar por que a participação social não pode surgir no setor educativo do México. Baseado em um trabalho empírico com escolas secundárias de três estados: Chiapas, Ciudad de México e Durango ( $n=27)$, este estudo evidencia que os indivíduos mostram predisposição a intervir de maneira crítica e fundamentada em seus centros escolares; não obstante, por não existirem estruturas de oportunidade para ampliar competências cidadãs, esses mesmos atores não parecem ser vistos como iguais, havendo entendimento superficial do mundo juvenil por parte dos professores, que impedem que a participação do cidadão se efetive, apesar do regime autoritário construído pelo sistema de partido único (1920-2000).

Palavras-chave: Igualdade. México. Participação cidadã. Escola secundária.
\end{abstract}

\section{Introducción}

En un estudio sobre política educativa, Pablo Latapí-Sarre (2004, p. 313), asienta que, en México, la participación de la sociedad ocurre en un contexto cultural y político en donde es visible "una ausencia de una cultura democrática debido al régimen autoritario y prácticamente monopólico". Para este analista, la pasividad de los gobernados se debe al paternalismo del Estado que "coarta el ejercicio de sus derechos ciudadanos formalmente reconocidos” (LATAPÍ-SARRE, 2004, p. 313).

Pero, iserá verdad que hay una ruta de dependencia con el pasado que no nos permite actuar de modo democrático dentro de las escuelas para mejorarlas? ¿Es tan fuerte El Ogro Filantrópico (PAZ, 1979) para no dejarnos desplegar la participación ciudadana? Este es el punto que abordaré en este artículo. El argumento central es que no necesariamente tenemos un problema de cultura, sino de falta de estructuras y reglas de participación dentro del espacio escolar. Esto se sostiene con los resultados de un estudio empírico realizado en 27 escuelas secundarias $(\mathrm{n}=27)$ de Chiapas, Ciudad de México y Durango.

El texto está dividio en tres partes principales. En la primera hablaré de algunas líneas de análisis de Latapí-Sarre (2004) y Zurita (2008; 2010) sobre la participación social. Específicamente, trataré de subrayar qué lecciones hay en la materia y qué vacíos surgen a partir de sus investigaciones. Segundo, haré un repaso de los estudios más recientes sobre la política de participación social tratando de ofrecer nuevos elementos para su análisis. En la tercera y última parte, hablaré de algunos hallazgos de la investigación empírica. 


\section{Los problemas para participar democráticamente}

México es una democracia joven. Apenas en el año 2000 cambió el régimen de partido único (El PRI, Partido Revolucionario Institucional) por una democracia basada en elecciones relativamente limpias y competidas.

La herencia que dejó el régimen autoritario del PRI sobre la cultura política de los mexicanos ha sido objeto de estudio de varios especialistas. En el ámbito de la educación, Latapí-Sarre (2004, p. 313) manifiestaba que la participación de la sociedad ocurre en un contexto cultural y político en donde es visible "una ausencia de una cultura democrática debido al régimen autoritario y prácticamente monopólico". Para él, la pasividad de los gobernados se debe al paternalismo del Estado que "coarta el ejercicio de sus derechos ciudadanos formalmente reconocidos".

En el advenimiento de la democracia, Observatorio Ciudadano de la Educación (OCE) (OCE, 1999), fundado por Pablo Latapí-Sarre, retomaba el tema de la participación ciudadana en la escuela, específicamente, a través de los Consejos Escolares de Participación Social (CEPS). Ahí OCE identificaba las causas de la inoperancia de los CEPS en la escuela mexicana.

1. la inadecuación del modelo propuesto en la ley general de educación a las circunstancias concretas del plantel,

2. la confusión entre sus atribuciones y las de las asociaciones de padres de familia,

3. las resistencias de algunos directores y maestros,

4. los intereses sindicales o partidistas,

5. la ausencia de hábitos de participación.

De estos cinco factores, dos de ellos hacían referencia al diseño institucional de la políticas, uno era una variable externa (la que se refiere al sindicato) y dos a la manera en que se comportan y actúan los agentes escolares. Para OCD (OCD, 1999), había resistencias de maestros y directivos, así como una ausencia de hábitos de participación.

Para autores como OCE y Latapí-Sarre, era urgente, "democratizar el poder sobre la educación” (LATAPÍ-SARRE, 2004) y esto lo intuían desde antes que la democracia se instaurara en México. En 1992, por ejemplo, Latapí-Sarre (1992) reafirmaba que "[1]a democracia no son sólo los episodios electorales, sino una relación activa entre gobernantes y gobernados en las decisiones cotidianas". 
Cuando uno repasa los factores que podrían explicar la débil participación social en México, uno también puede hallar otra clasificación. Aquellos que tienen que ver con la "oferta", es decir, los que parten desde el gobierno y los que están circunstritos en la sociedad ("demanda"). Entre estos, podemos encontrar la falta de "hábitos de participación" de los padres de familia o las "resistencias" de los maestros y directivos (LATAPÍ-SARRE, 2004).

Pese a la utilidad de esta clasificación (“oferta y demanda”), no podemos decir que ambas dimensiones están completamente disociadas, más bien la pregunta es cómo se recrean en una relación funcional: si el gobierno sofoca el florecimiento ciudadano, los hábitos de participar pueden menguar y viceversa, si ciudadanos no presionan a los gobiernos, éstos no tendrán incentivos para crear una "institucionalidad democrática". Sobre esta base se tratará de construir una nueva explicación sobre el desarrollo de la política de participación social.

Pero pasemos ahora a la segunda parte del texto. ¿Qué se ha dicho sobre la participación social en la escuela y qué lecciones podemos recoger para emprender nuevos estudios en la materia?

\section{Qué se sabe sobre participación social²}

El estudio de la participación social en México se ha desarrollado en los últimos años. Incluso, se fundó, una red de investigadores sobre participación social que está compuesta por colegas de distintos centros de estudios y universidades públicas del país.

Una de estas colegas es Úrsula Zurita (2008; 2010), quien ha conducido dos estudios sobre la participación social en las escuelas de México. Una de ellas, tuvo un universo amplio y se intituló, Evaluación Nacional de la Participación Social en la Educación Básica 2000-2006 mientras que la segunda, fue regionalmente acotada ya que tomó el caso de la Ciudad de México.

En la primera investigación, se analiza la participación en los tres niveles de educación básica (preescolar, primaria y secundaria) al igual que en varias de sus modalidades (general, indígena y comunitaria). Las preguntas que guiaron el estudio de Zurita fueron cómo los actores escolares viven la participación social, cómo la valoran y cuáles son sus expectativas sobre ella. Es decir, se centró en los atributos de la "demanda".

Uno de los hallazgos más sobresalientes fue que la política de participación sí ha permeado, discursivamente, a las escuelas y se le valora positivamente. Pese a 
ello, Zurita (2008) aclara que prevalecen sentimientos de temor, desconfianza y rechazo de la participación social. Hay, por lo tanto, una ambivalencia de los actores escolares sobre el tema.

Otro punto que detecta Zurita (2008) es que los padres no saben en qué actividades participar, pero éstos no se duermen en sus laureles y demandan también capacitación, información, apoyo, acompañamiento y asesoría para impulsar la PS. Este hallazgo nos muestra que sí parece haber una predisposición a participar, la cultura vertical del régimen político quizás ya no permea lo suficiente a los padres de familia para inmovilizarlos, sin embargo, éstos no encuentran la "estructura de oportunidades para ampliar sus capacidades ciudadanas”. Explicaré más adelante este punto.

Participar activamente en la escuela o en la comunidad demanda habilidades y destrezas que cuando se echaron los consejos de participación, se dio por sentado que las poseíamos. La Secretaría de Educación Pública (SEP) pudo ser entonces un "pedagogo democrático", cosa que no oucrrió.

Posterior al estudio de la Evaluación Nacional, Zurita en 2010 concentra su atención en un solo nivel educativo: el de primaria. Ahí, recaba información, mediante una encuesta, con cuatro actores escolares: (1) supervisores, (2) directores, (3) maestros y (4) padres de familia. Para Zurita, sí hay participación social, pero de manera heterogenea y diferente a lo que se plantea en los modelos oficiales.

Este hallazgo muestra por un lado, que sí hay "inadecuación" entre el modelo oficial de PS y la realidad de las escuelas, como señaló Observatorio hace más diez años; pero por otro, vuelve a enfatizar que los actores sociales no son pasivos, sí están involucrándose en los asuntos escolares. Hay entonces un matiz para no suscribir automáticamente la idea de los Ciudadanos imaginarios (ESCALANTE, 1993).

Otra observación de Zurita (2010) es que la inclusión de los estudiantes como protagonistas de la PS es inexistente. ¿Bajo qué idea se prescinde del niño o del joven en las discusiones para mejorar la vida escolar? ¿Será que la política de participación social sigue impregnada del paternalismo clásico del antiguo régimen? ¿No ve el gobierno a los niños y a los jóvenes como individuos capaces de actuar razonadamente?

Sobre este punto, es muy interesante que Zurita (2010) haya encontrado que existe un temor por parte de directores y maestros de "politizar" la escuela si incluyen a más miembros, cuestión que no ocurre ni en Brasil ni en Perú, según otros estudios. 
Pareciera que en las escuelas hay temor de discutir asuntos escolares, ¿por qué?, ies esto un reflejo del autoritarismo político instaurado por el régimen del partido único? Latapí-Sarre (2004, p. 313-314) suscribe la fuerza de la cultura autoritaria del régimen político sobre el actuar pasivo de las personas, no obstante, también intuye algo importante: "Toda cultura política se construye a partir de prácticas institucionales". Esto punto es clave para avanzar la explicación en la materia de las políticas públicas. Con su intuición, Latapí-Sarre nos ayuda a pensar cómo las diversas perspectivas del nuevo institucionalismo (NORTH, 1995) podrían explicar las fallas de la política de la participación social en la escuela y en el campo de la política educativa de México. Con esta perspectiva, hay posibilidades de rebasar las "tesis culturalistas". No todo lo explica la "cultura" dado que los individuos podemos negociar nuestras tradiciones y crear, mediante la práctica, reglas distintas de las que "vienen" del pasado. Sobre esta basa de interpretación social es que se construyen los argumentos de este texto. Pero sigamos con la revisión de literatura.

El OCE observaba que a pesar de que el gobierno anunciara la instalación multitudinaria de consejos escolares (CEPS), no había evidencia de su funcionamiento. Esta situación cambio. La SEP, en 2012, condujo un estudio para crear indicadores del "ciclo funcional" de los CEPS. Basándose en el Registro Público de Consejos Escolares, la SEP estableció cuatro etapas de dicho ciclo, a saber:

a) Conformación: abarca la integración del Consejo Escolar, el nombramiento de sus diferentes cargos, las instalaciones de los comités y la planeación de las actividades que realizará durante el ciclo escolar.

b) Gestión: se refiere a la operación de los consejos.

c) Evaluación: orientada a explorar los resultados que obtiene el consejo escolar en relación a las actividades y metas puntuales.

d) Transparencia y rendición de cuentas: los CEPS cumplen con una función de transparencia en tanto pone a disposición pública la información generada en las sesiones y asambleas (SECRETARÍA DE EDUCACIÓN PÚBLICA, 2012, p. 14).

Los resultados muestran que a mayor complejidad de las funciones de los consejos, menores instancias de este tipo se involucran. Por ejemplo, en la fase de conformación, se dijo que existían 192,115 CEPS registrados. Esto representa 79,5 por ciento del total de los centros escolares del país, de educación inicial, especial y básica. Otro hallazgo interesante es que los servicios indígenas de preescolar y pri- 
maria, superan por mucho el promedio nacional de cobertura en un 98 por ciento y 97,4 por ciento respectivamente, lo que hace pensar que en zonas de mayor desventaja se logran instaurar en mayor medida los esquemas formales de participación social (SECRETARÍA DE EDUCACIÓN PÚBLICA, 2012).

En la segunda fase (gestión), la SEP (2012) reporta que sólo 14 por ciento de los CEPS realizan tareas como la "integración de comités temáticos, búsqueda de programas educativos de apoyo y determinación de metas con base en los resultados de la prueba Enlace" (SECRETARÍA DE EDUCACIÓN PÚBLICA, 2012, p. 46). En esta fase, llamo la atención que sólo uno por ciento de los CEPS expresaba tener algún tipo de vinculación con el Consejo Municipal o Estatal de Participación Social, lo que podría significar que la escuela y la comunidad no parecen estar interrelacionadas.

Por otra parte, en la tercera fase (evaluación), se reporta que sólo 14 por ciento de los CEPS estableció metas para mejorar los resultados de Enlace.

Por último, en la fase de transparencia, el informe sobre los indicadores revela que del total de CEPS conformados, sólo ocho por ciento reportó conocer el plan de trabajo de la escuela, lo que representa un total de 16,002 consejos. Aún más preocupante es que solo 0,3 por ciento del total de los CEPS reportó información para generar un informe de transparencia y rendición de cuentas (SECRETARÍA DE EDUCACIÓN PÚBLICA, 2012).

En síntesis, los resultados de las fases de los consejos escolares, no son alentadores. Entre más complejas son las atribuciones de los CEPS, un menor número de ellos realiza tales funciones. Esto indica la necesidad de no dar por sentado que tenemos conocimientos y habilidades para participar. Habrá que desarrollarlas y en ello, la SEP tendría que reconocerse como un "pedagogo democrático" que en primer lugar, no le tenga temor a la participación y para ello, reconozca a los agentes escolares como responsables de su actuación y que proponga una "estructura de oportunidades" para ampliar las competencias ciudadanas.

Para reforzar este punto, se puede decir que la literatura sobre la participación social en la escuela muestra que a medida que se transfieren mayores responsabilidades a los actores educativos, se potencia la intervención y se extienden las posibilidades de decisión colectiva. En algunas áreas de Brasil, por ejemplo, no hay miedo ni temor - como en el caso mexicano, ver Zurita (2008) - a que los actores tengan atribuciones complejas tales como elegir a sus directivos. Al elegir a los directivos, según Leite et al. (2012), se reduce la brecha entre la parte administrativa (dirección) y la parte pedagógica (coordinadores). 
En resumen, la participación parece no surgir de manera automática o espontánea, hay que cultivar las bases para desplegar capacidades ciudadanas y de participación democrática y aquí la SEP tiene una responsabilidad como "pedagogo democrático".

Hasta aquí, se han resaltado al menos cuatro lecciones de la literatura revisada. Primero, los estudios empíricos muestran que la ausencia de participación ciudadana no está del todo presente. Los sujetos participan de una u otro manera y de manera heterogenea como apunta Zurita (2010). La pasividad de los agentes escolares, como aparece en los recientes estudio, tendrá que ser revisada en el futuro.

Segundo, al echar a andar los CEPS, se dio por sentado que los mexicanos teníamos las habilidades para participar en las comunidades y en las escuelas, sin embargo, son los propios actores como los padres de familia, los que demandan capacitación para poder hacerlo. Esto levanta la necesidad de pensar - una vez más - en un "pedagogo democrático" que nazca desde la SEP, aunque la duda que me invade a este respecto es a qué grado una oficina del gobierno puede promover de modo genuino la participación ciudadana.

Tercera observación: parece prevalecer una visión estrecha sobre quiénes son los niños y jóvenes y cómo podrían participan en la vida escolar. Esta estrecha visión sigue presente. Es sintomático que la SEP pretende formar niños y jóvenes que conozcan las reglas de convivencia, que aporten sus habilidades para el trabajo colectivo, que actúen con apego a los valores democráticos y que dialoguen para solucionar conflictos, pero que no estén, ni los niños ni los jóvenes, considerados como sujetos actuantes y con voz dentro de los CEPS.

Cuarta y última observación recogida de la revisión de literatura: entre más libertades existan para las escuelas y sus agentes escolares, la participación social puede irse acrecentando. Esto aún se mira con resquemor por parte de la SEP, a pesar de que la Ley General de Derechos de las Niñas, Niños y Adolescentes, estipula que la libertad de expresión conllevará el derecho a que se tome en cuenta su opinión respecto de los asuntos que les afecten directamente, a sus familia o comunidades (Artículo 64).

Entonces, ¿quién le teme al empoderamiento de los agentes escolares? ¿Las grandes burocracias como la SEP y el Sindicato Nacional de Trabajadores de la Educación (SNTE)? Con respecto a este último actor, vale la pena decir que ningún estudio reseñado en nuestro estudio habló del SNTE como un impedimento para la participación social en la escuela. Esto resulta extraño, ya que en su libro la $S E P$ por dentro, Latapí-Sarre (2004) recoge argumentos de ex secretarios de Educación 
Pública que apuntaron hacia una oposición "histórica" del sindicato a esta política. ¿Será que ya desistieron de pelear este punto? No se sabe y mayor información es necesario. Pasemos ahora a presentar los resultados de nuestra investigación empírica.

\section{Participación en la secundaria: dos mundos ${ }^{3}$}

La pregunta que guió esta investigación fue: ¿a qué grado los agentes escolares son capaces de promover la participación social a pesar de haber padecido un régimen político vertical y corporativista? Para ello, acordamos una metodología basada en tres etapas:

1. Elegimos el nivel de secundaria, dado que es este tramo de la educación básica obligatoria la que presenta, según los estudios del INEE, los mayores problemas en términos de aprendizaje. Es la secundaria de México la que alimenta el rezago educativo, el cual como ustedes saben rebasa los 33 millones de personas. Además, estudios sobre la participación en la primaria son más comunes.

2. Nos concentramos en las secundarias públicas generales en tres estados de las República Mexicana: Chiapas, Durango y Ciudad de México. Estos estados los elegimos por medio de un criterio regional y de diversidad cultural. Luego seleccionamos diez escuelas por cada estado de acuerdo con dos criterios: desempeño académico según la prueba Enlace (nivel alto, medio, bajo) y si tenían instalado en consejo de participación social. Al final tuvimos acceso a 27 centros escolares.

3. La información la recabamos mediante dos instrumentos: una encuesta a los directores y una entrevista colectiva (grupos de enfoque) a estudiantes, maestros y padres de familia. Con el propósito de llenar los vacíos que advertimos en la revisión de literatura, elegimos a los estudiantes como informantes clave y no sólo a los padres, maestros y directores como se había hecho en la mayoría de las investigaciones.

4. ¿Qué resultados obtuvimos? Primero, ante la pregunta de si los estudiantes eran sujetos participativos, los maestros, de las diversas escuelas de los tres estados, coincidieron en tener una visión bastante pesimista de los jóvenes estudiantes. Los tachan de "apáticos" a pesar, dicen ellos, de tener más recursos que las pasadas generaciones. 
Una maestra de la Ciudad de México afirmó que los jóvenes estudian porque los obligan y otra de Chiapas, remató diciendo que están faltos de valores y por eso hay muchos problemas como el del consumo de alcohol y drogas. Cuando se preguntó sobre qué factores puede producir esas supuestas actitudes, los docentes de Durango y Chiapas culparon directamente a lo que ocurre en la casa. El siguiente testimonio es revelador en varios sentidos:

Hace años venía en los códigos que se debía castigar al padre si el niño delinquía [...] la responsabilidad del hijo es del padre no del maestro, porqué yo voy a ser maestra de [ese] hijo por dos o tres años [mientras] que él [el padre] va a ser toda la vida.

¿Debe el Estado castigar al padre si el joven delinque? ¿No debería ser el adolescente responsable de sus actos? La responsabilidad sobre nuestras acciones nos hace ciudadanos y en las las democracias liberales se busca precisamente este objetivo: crear sujetos responsables de sus actos.

Otro de los "villanos favoritos" de la aparente falta de valores de los jóvenes - que en realidad, eran valores de los adultos - era la tecnología. Un profesor de Chiapas aseguró que la televisión, el internet y el celular es "su mundo" de los chicos y cuesta trabajo que aterricen en su clase. ¿Será que a los jóvenes no les interesa estudiar, aprender y obtener conocimiento?

Con ese visión pesimista de la juventud, iniciamos las entrevistas colectivas a los jóvenes de entre 12 y 15 años. ¿En verdad son los individuos que piensan sus maestros? ¿Está perdida la causa de la participación social si confiaramos en los jóvenes? Al preguntarles a los muchachos en una escuela de Chiapas, qué es la democracia, obtuvimos los siguientes testimonios:

[La democracia es] la participación, la opinión o la expresión que nosotros hacemos para dar un puntos de vista, es decir, la democracia [se piensa] que es votar, pero en mi punto de vista, no sólo es votar, sino tomar decisiones tanto en nuestra vida interior como exterior.

Otro joven complementó esta idea diciendo que la democracia se piensa como aspectos del mero gobierno, pero no, también, según él, tiene que ver con nosotros”.

$\mathrm{Al}$ rebasar la noción clásica de democracia representativa, los jóvenes coinciden con Latapí-Sarre (2005, p. 47) cuando expresa que la democracia no consiste sólo en "episodios electorales", sino en una "relación activa entre gobernantes y gobernados en las decisiones cotidianas".

Pero en nuestra investigación, no sólo quisimos indagar cómo conceptualizaban los estudiantes la democracia, sino que también nos propusimos explorar cómo estas ideas podían realizarse en la vida cotidiana y escolar de los jóvenes. Para ello, tratamos de observar ocho competencias ciudadanas. Estas eran: 
1. Identificación de problemas escolares.

2. Capacidad crítica

3. Asumir responsabilidad

4. Expresión pública de los problemas

5. Defensa de argumentos propios

6. Búsqueda de soluciones

7. Diálogo abierto

8. Capacidad para actuar colectivamente.

Gracias a los grupos de enfoque, pudimos corroborar algunas de estas competencias en las distintas escuelas de los tres estados seleccionados. En un ejercicio primario de triangulación de testimonios, pudimos constatar la inconformidad de los jóvenes con diversas condiciones de sus escuelas. En San Cristóbal de las Casas, Chiapas, un joven expresó, "no tenemos espacio [...] ni recursos para construir una escuela [...] sólo hay dos maestros y nos toca pintar las paredes para poder estar aquí [además] tenemos que contribuir a comprar las escobas".

El nivel de exigencia académica de los jóvenes fue patente también en la Ciudad de México donde un muchacho expresó. Ante la pregunta de cuáles son los problemas de su centro escolar, un joven describió los problemas que tenía con un maestro de Historia. Dijo:

según es abogado y casi nunca viene y cuando llega a venir nada más se la pasa diciendo que nos portemos bien, nunca nos da clases. Nada más nos deja estar copiando en el libro y no nos enseña nada, me cae mal.

El testimonio del joven de Chiapas es muy interesante, ya que se quejó de una actividad que ha sido tomada recurrentemente como expresión de la participación social y esto es que se mejore la infraestructura escolar. Pocos nos negaríamos a que los jóvenes embellezcan sus espacios de estudio, el punto aquí es que el estudiante logró identificar un problema mayor y que es no tener espacios de estudio, ni al patio podían salir a jugar, expresó.

En el caso del joven de la Ciudad de México, es interesante porque luego de quejarse de las ausencias de su profesor y de su manera de dar clases, emite un juicio: "me cae mal" porque no enseña nada. Este testimonio contrasta con la visiones pasivas y conformistas que mantenían algunos profesores de los propios jóvenes. ¿Será que el profesor y el estudiante no se están entendiendo?

Nuestro estudio (RAMÍREZ, A., 2015; RAMÍREZ, L., 2015) mostró una amplia brecha entre lo que son en realidad los jóvenes y lo que expresaron de ellos sus 
profesores. Bajo esta premisa, ¿podría desencadenarse procesos de participación activa y colectiva? Dejo este punto abierto para tratarlo en las conclusiones, mientras sigamos repasando algunos testimonios de los jóvenes que dan cuenta de sus competencias democráticas.

Como mencioné arriba, asumir la responsabilidad de nuestras acciones nos hace mirarnos como individuos. Nosotros y no la corporación es la que decide qué hacer y esto acarrea consecuencias para los otros.

$\mathrm{Al}$ preguntarles a los jóvenes sobre qué hacen para tener éxito en la escuela expresaron lo siguiente: "Los profesores y padre de familia están involucrados en ello, pero también depende de nosotros 'más que nada', porque si tú no buscas la manera y el apoyo [no lo lograrás]”. En Durango, una secundaria introdujo, por iniciativa del director, un programa orientado a la disciplina con el tema de la autorregulación. Ahí, los jóvenes expresaron que era útil porque podían controlar sus acciones y no estar a expensas de que te digan qué debes hacer.

Ya se ha dicho que los jóvenes parecen ser conscientes de la problemática de sus escuelas y se expresan abiertamente. Asimismo, en el breve repaso de testimonios tampoco se ve que valoren positivamente la ausencia de los maestros "barco" y sus métodos arcaicos de enseñanza. También hemos dicho que parecen hacerse responsable de sus actos, pero la pregunta es: ibuscan soluciones en conjunto a sus problemas para poder defender que sean miembros de los CEPS?

Fue muy interesante constatar que los jóvenes sí actuaban en referencia de varios temas que les afectaban sensiblemente como el hostigamiento escolar y otros que eran más de consciencia ecológica. En Durango, por ejemplo, nos contaron que hubo un problema de venta de drogas dentro de la secundaria y que ahora ya no es así. La solución fue que ellos mismos hablaron con el drugs dealer para pedirle que el negocio lo hiciera afuera del centro escolar, de lo contrario hablarían con los maestros. Pese a lo preocupante de tal situación, se puede decir que los jóvenes resolvieron un problema dentro de su espacio escolar y se lo trasladaron a otros actores (policía, sociedad) que debería cuidar, en teoría, lo que ocurre fuera de las escuelas.

Retomo ahora el punto de la tensión entre la labor del maestro y las actitudes de los estudiantes. Es muy pertinente por los tiempos que vivimos discutir el equilibrio de poder que tienen tanto profesores como estudiantes dentro de la escuela mexicana y si tal situación es benéfica.

Sin visiones candidas de ningún lado, hay que analizar si el relajamiento de la disciplina y la falta de valores que observan los maestros no es más que una mutación de la cultura juvenil. Me explico: los jóvenes parecen tener márgenes de 
libertad más amplios por la manera en que hoy vivimos y esto puede hacerlos más reactivos, seguros, conscientes de sus potencialidades, con mayores riesgos pero también más individuos.

Sobre el desequilibrio de poder en la escuela, los jóvenes decían que ellos llevaban las de perder si hay un problema con un profesor. Una estudiante de Durango dijo lo siguiente:

una maestra con la que tuvimos un problema nos trajo a su papá que era el supervisor de toda la zona - o algo así. El señor nos dijo, "levántense el que quiera respetar a la maestra" y la mayoría no se levantó. [Luego] nos preguntaron que por qué y una niña preguntó: ¿Por qué ella no nos respeta a nosotros también? Y entonces la maestra hizo su cara de "claro que no" [los respeto]. Cuando se fue el profe, dijo que no le interesaba lo que fueran a decir, que su papá podía hacer lo que ella quisiera.

Ante las ínfulas de la maestra, enfrentar a un profesor como lo hizo esa jovencita de Durango, alienta. Es un pequeño gesto que me hace pensar en lo grande que puede ser la independencia de la persona ante el poder.

Una de las conclusiones de nuestro estudio es que mientras no haya una consciencia y una manera concreta de mirarse como iguales profesores y estudiantes, será muy difícil propiciar procesos de participación social.

Quizás esta observación pueda enriquecerse con la ayuda de George Homans (1961 apud ALEXANDER, 1990), quien observó que un grupo de personas que convive y se interrelaciona constantemente puede llegar a desarrollar una cultura que antes no existía y además, puede acatar ciertas normas. No obstante, este principio tiene una salvedad, como explica Randall Collins (1975 apud ALEXANDER, 1990), de que el proceso de grupo se produce solamente cuando sus miembros parten de un plano de igualdad. Vaya reto, sentirnos iguales para interactuar, participar y cooperar.

Entonces, no crear ni sentirse dentro de condiciones de igualdad parece entorpecer la participación ciudadana. ¿Por qué? Porque es difícil que uno acepte que alguien de menor jerarquía establezca lo que está bien o mal, mucho menos que te diga qué hacer para mejorar el proceso educativo.

\section{Reflexiones finales}

Uno de los argumentos centrales de este artículo fue que, a partir del trabajo empírico, se analice la participación social en la escuela bajo un encuadre analítico que sea capaz de integrar los factores gubernamentales que impiden el surgimiento de la ciudadanía en la escuela con aquellos de los propios agentes escolares. De 
esta manera podríamos rebasar las tesis culturalistas en los que se asientan ciertas explicaciones sobre las fallas de la política de participación ciudadana.

Aunque a nivel macro, la participación no electoral en México parece ser, en general, desestructurada (IFE, 2014), diversos estudios empíricos recientes han mostrado que también es visible una ciudadanía "inesperada", como bien lo detectaron Ariadna Acevedo y Patricia López (2012).

En este estudio se cuestionó la idea de que no hay participación ciudadana en la escuela porque una determinada "cultura política" nos lo impide. Hay esfuerzos, iniciativas y voces que no encuentran eco en una estructura formal de participación. Sigue habiendo, por lo tanto, “inadecuación” entre lo oficial y lo real y para poder sobre pasar esta situación no se necesitan hacer más lineamientos cada año de la operación de los consejos escolares. El reto es mayúsculo.

Crear un "pedagogo democrático" desde la SEP consistiría en imaginar y abrir oportunidades para que los niños, jóvenes y sus padres de familia amplían sus competencias ciudadanas. Es precisamente desde la "desarticulada” pero espontánea participación desde donde se deben planear los cambios. Uno de estos cambios sería promover planos de igualdad entre maestros y estudiantes para que pueda haber interrelación grupal, se compartan normas comunes y se avance en lo social.

Quizás al decir esto estamos ahora frente a dos anhelos inseparables la construcción ciudadana para desarrollar nuestra débil democracia y la igualdad de libertades y recursos para poder constituir la justicia social. El debate está abierto.

\section{Notas}

1 Esta investigación fue financiada por el Conacyt y la realicé junto con Ana V. Ramírez y Laura Ramírez.

2 Este apartado se basa en el artículo La participación social en la escuela en México que escribí con Ana. V. Ramírez Ramón (FLORES-CRESPO; RAMÍREZ RAMÓN, 2015).

3 Esta parte la escribí con base en la tesis de maestría de Laura Ramírez (2015).

\section{Referencias}

ACEVEDO, A.; LÓPEZ, P. (coord.). Ciudadanos Inesperados. Espacios de Formación de Ciudadanía ayer y hoy. México: Colmex-Cinvestav, 2012.

ALEXANDER, J. Las teorías sociológicas desde la Segunda Guerra Mundial. México: Gedisa, 1990.

FLORES-CRESPO, P.; RAMÍREZ RAMÓN, A. V. La Participación Social en la Escuela en México. Una revisión de literatura. Revista Iberoamericana sobre Calidad, Eficacia y Cambio en Educación, España, v. 13, n. 3, p. 77-94, 2015. Disponible en: http://www.rinace.net/reice/numeros/arts/vol13num3/art5.pdf. Acceso en: 26 ago. 2019. 
ESCALANTE, P. Ciudadanos imaginarios: memorial de los afanes y desventuras de la virtud y apología del vicio triunfante en la República Mexicana: Tratado de moral pública. México: Colmex, 1993.

IFE. Informe País Sobre la Calidad de la Ciudadanía en México. México: Instituto Federal Electoral, Colegio de México, 2014.

LEITE, Maria Cecilia Lorea et al. Gestión escolar democrática: una construcción contextualizada en escuelas municipales de la ciudad de Pelotas, RS, Brasil. RMIE, México, v. 17, n. 52, p. 89-113, mar. 2012. Disponible en: http://www.scielo.org.mx/scielo.php?script=sci_ arttext\&pid=S1405-66662012000100005\&lng=es\&nrm=iso. Acceso en: 26 jun. 2019.

NORTH, D. C. Instituciones, cambio institucional y cambio económico. México. FCE, 1995.

OCE. Comunicado 1: ¿Qué pasa con los Consejos de Participación Social? México: OCE, 1999.

PAZ, O. El Ogro Filantrópico: historia y política, 1971-1978. México: J. Mortiz, 1979.

RAMÍREZ, L. Actitudes democráticas para la participación social en jóvenes de secundarias generales. Tesis (Maestría en Investigación y Desarrollo Educativo) - UIA, México, 2015.

RAMÍREZ, A. V. Participación social, gestión y democracia: dificultades y avances en la secundaria de México. Tesis (Maestría en Investigación y Desarrollo Educativo) - UIA, México, 2015.

LATAPÍ-SARRE, P. ¿Quién tendrá el poder sobre la educación? In: LATAPÍ-SARRE, P. Tiempo Educativo Mexicano I. México: UAA, 1992.

LATAPÍ-SARRE, P. La SEP por dentro: las políticas de la Secretaría de Educación Pública comentadas por cuatro de sus secretarios. México: Fondo de Cultura Económica, 2004.

LATAPÍ-SARRE, P. La participación de la sociedad en la definición de políticas públicas de educación: el caso de Observatorio Ciudadano de la Educación. Perfiles Educativos, México, v. 27, n. 107, p. 7-39, 2005.

SECRETARÍA DE EDUCACIÓN PÚBLICA. Indicadores del ciclo funcional de los consejos escolares de participación social ciclo escolar 2011-2012. México: Gobierno Federal, 2012.

ZURITA, U. Reflexiones en el marco de la Evaluación Nacional de la Participación Social en la Educación Básica 2000-2006. In: OBSERVATORIO CIUDADANO DE LA EDUCACIÓN. Participación social en la educación: de análisis a las propuestas. México: Observatorio Ciudadano de la Educación A.C., 2008. p. 63-78.

ZURITA, U. La educación para la vida democrática a través de la participación social: puntos de encuentro entre la escuela y la familia. Revista interamericana de educación para la democracia, Flacso, México, v. 3, n. 2, p. 171-194, 2010. 